\title{
FROM JANTZEN TO ANDERSEN FILTRATION VIA TILTING EQUIVALENCE
}

\author{
JOHANNES KÜBEL*
}

\begin{abstract}
The space of homomorphisms between a projective object and a Verma module in category $\mathscr{O}$ inherits an induced filtration from the Jantzen filtration on the Verma module. On the other hand there is the Andersen filtration on the space of homomorphisms between a Verma module and a tilting module. Arkhipov's tilting functor, a contravariant self-equivalence of a certain subcategory of $\mathcal{O}$, which maps projective to tilting modules induces an isomorphism of these kinds of Homspaces. We show that this equivalence identifies both filtrations.
\end{abstract}

\section{Introduction}

Let $\mathfrak{g} \supset \mathfrak{b} \supset \mathfrak{h}$ be a semisimple complex Lie algebra with a Borel and a Cartan. In BGG-category $\mathscr{O}$, prominent objects are indecomposable projective and tilting modules.

Tilting modules were introduced in [4] as selfdual Verma flag modules and the indecomposable tilting modules are classified by their highest weight. Let $\rho \in \mathfrak{h}^{*}$ be the halfsum of positive roots relative to $\mathfrak{b}$ and let $T$ denote the ring of regular functions on the line $\mathrm{C} \rho$. Then $T$ is a quotient of the universal enveloping algebra of $\mathfrak{h}$. For every weight $\lambda \in \mathfrak{h}^{*}$ the quotient map $\mathfrak{b} \rightarrow \mathfrak{h}$ induces a $\mathfrak{b}$-module structure on $\mathfrak{C}$. We denote this $\mathfrak{b}$-module by $\mathrm{C}_{\lambda}$. The $\mathfrak{h}$ module structure on $T$ also lifts to a $\mathfrak{b}$-module structure by the map $\mathfrak{b} \rightarrow \mathfrak{h}$. Now we can form the Verma module $\Delta(\lambda)=U(\mathfrak{g}) \otimes_{U(\mathfrak{b})} \mathrm{C}_{\lambda} \in \mathfrak{g}$-mod and the deformed Verma module

$$
\Delta_{T}(\lambda)=U(\mathfrak{g}) \otimes_{U(\mathfrak{b})}\left(\mathrm{C}_{\lambda} \otimes T\right) \in \mathfrak{g}-\bmod -T
$$

where tensor products without any specification are to be understood over $C$. The $T$-module structure on $\Delta_{T}(\lambda)$ is just multiplication from the right while the $\mathfrak{b}$-module structure on $\mathrm{C}_{\lambda} \otimes T$ is the tensor product representation.

\footnotetext{
*I would like to thank Wolfgang Soergel and Peter Fiebig for many helpful discussions and for teaching me the necessary tools to write this article. I am also grateful to the referees for their valuable suggestions.

Received 9 December 2010.
} 
Taking the direct sum of the $T$-dual weight spaces and twisting the contragredient $\mathrm{g}$-module structure with a Chevalley automorphism leads to the deformed dual Verma module $\nabla_{T}(\lambda) \in \mathfrak{g}$-mod- $T$. We will see that every invertible $T$-module homomorphism on the $\lambda$-weight spaces extends to an injective homomorphism of $\mathfrak{g}-T$-bimodules

$$
\text { Can }: \Delta_{T}(\lambda) \hookrightarrow \nabla_{T}(\lambda)
$$

which forms a basis of the $T$-module $\operatorname{Hom}_{\mathfrak{g}-T}\left(\Delta_{T}(\lambda), \nabla_{T}(\lambda)\right)$. Since $T$ can be understood as a polynomial ring in one variable $v$ we get the Jantzen filtration on $\Delta(\lambda)$ by taking the images of $\mathrm{Can}^{-1}\left(\nabla_{T}(\lambda) v^{i}\right)$ for $i=0,1,2,3, \ldots$ under the surjection $\Delta_{T}(\lambda) \rightarrow \Delta(\lambda)$ induced by $\cdot \otimes_{T}$ C. For a projective object $P \in \mathcal{O}$ we get an induced filtration on $\operatorname{Hom}_{\mathfrak{g}}(P, \Delta(\lambda))$.

Let $\widehat{T}$ be the completion of $T$ at the maximal ideal of 0 . So we can identify $\widehat{T}$ with the ring of formal power series $C \llbracket v \rrbracket$ in one variable. In this article, we will also introduce deformed tilting modules which are certain $\mathrm{g}-\widehat{T}$-bimodules corresponding to tilting modules in $\mathscr{O}$ after specializing with $\cdot \otimes_{\widehat{T}} \mathrm{C}$. Now let $K$ be such a deformed tilting module and consider the composition pairing

$$
\operatorname{Hom}\left(\Delta_{\widehat{T}}(\lambda), K\right) \times \operatorname{Hom}\left(K, \nabla_{\widehat{T}}(\lambda)\right) \longrightarrow \operatorname{Hom}\left(\Delta_{\widehat{T}}(\lambda), \nabla_{\widehat{T}}(\lambda)\right) \cong \widehat{T}
$$

where all Hom-spaces are meant to be homomorphisms of $\mathrm{g}-\widehat{T}$-bimodules. We will see that this is a nondegenerate pairing of free $\widehat{T}$-modules of finite rank and leads to an injection

$$
\operatorname{Hom}\left(\Delta_{\widehat{T}}(\lambda), K\right) \hookrightarrow\left(\operatorname{Hom}\left(K, \nabla_{\widehat{T}}(\lambda)\right)\right)^{*}
$$

where $(\cdot)^{*}$ denotes the $\widehat{T}$-dual.

Taking the preimages of the $\widehat{T}$-submodules $\left(\operatorname{Hom}\left(K, \nabla_{\widehat{T}}(\lambda)\right)\right)^{*} \cdot v^{i}$ under this embedding and applying $\cdot \otimes_{\widehat{T}} \mathrm{C}$ to these preimages defines the Andersen filtration on $\operatorname{Hom}_{\mathfrak{g}}\left(\Delta(\lambda), K \otimes_{\widehat{T}} \mathrm{C}\right)$.

In [10], Soergel introduces the tilting functor $t$ which forms a contravariant self-equivalence of the category of modules with a Verma flag, i.e., a filtration with subquotients isomorphic to Verma modules. The functor $t$ takes projective modules to tilting modules and sends a Verma module $\Delta(v)$ to the Verma module $\Delta(-2 \rho-v)$. So $t$ induces an isomorphism of vector spaces

$$
\operatorname{Hom}_{\mathfrak{g}}(P, \Delta(\lambda)) \stackrel{\sim}{\longrightarrow} \operatorname{Hom}_{\mathfrak{g}}(\Delta(-2 \rho-\lambda), t(P))
$$

which we denote by $t$ as well. In this paper we will prove that $t$ identifies the filtration induced by the Jantzen filtration on the left side with the Andersen filtration on the right side. 
In [11], Soergel uses a hard Lefschetz argument to prove that the Andersen filtration on $\operatorname{Hom}_{\mathfrak{g}}(\Delta(\lambda), K)$ for $K$ a tilting module coincides with the grading filtration induced from the graded version of $\mathcal{O}$ as described in [3]. Since this is very similar to the result in [2] about the semisimplicity of the subquotients of the Jantzen filtration, the relation of both filtrations might give an alternative proof of this semisimplicity.

\section{Preliminaries}

This section contains some results about the deformed category $\mathscr{O}$ of a semisimple complex Lie algebra $\mathfrak{g}$ with Borel $\mathfrak{b}$ and Cartan $\mathfrak{h}$, which one can also find in [5] and [11]. By $S$ we will denote the universal enveloping algebra of the Cartan $\mathfrak{h}$ which is equal to the ring of polynomial functions $C\left[\mathfrak{b}^{*}\right]$. Let $T$ be a commutative, associative, noetherian, unital, local $S$-algebra with structure morphism $\tau: S \rightarrow T$. We call $T$ a local deformation algebra.

In this article we will mostly deal with the $S$-algebras $R=S_{(0)}$, the localization of $S$ at the maximal ideal of $0 \in \mathfrak{h}^{*}$, localizations $R_{\mathfrak{p}}$ of $R$ at a prime ideal $\mathfrak{p}$ of height 1 or the residue fields of these rings $\mathrm{K}_{\mathfrak{p}}=R_{\mathfrak{p}} / R_{\mathfrak{p}} \mathfrak{p}$. To apply results of this section to both filtrations we will also be concerned with the power series ring $C \llbracket v \rrbracket$ in one variable and the quotient field $Q$ of $S$. All these rings are local deformation algebras.

\subsection{Deformed category $\mathcal{O}$}

Let $T$ be a local deformation algebra with structure morphism $\tau: S \rightarrow T$ and let $M \in \mathfrak{g}$-mod- $T$. For $\lambda \in \mathfrak{h}^{*}$ we set

$$
M_{\lambda}=\{m \in M \mid h m=(\lambda+\tau)(h) m \forall h \in \mathfrak{h}\}
$$

where $(\lambda+\tau)(h)$ is meant to be an element of $T$. We call the $T$-submodule $M_{\lambda}$ the deformed $\lambda$-weight space of $M$.

We denote by $\mathcal{O}_{T}$ the full subcategory of all bimodules $M \in \mathrm{g}$-mod- $T$ such that $M=\bigoplus_{\lambda \in \mathfrak{h}^{*}} M_{\lambda}$ and with the properties that for every $m \in M$ the $\mathfrak{b}-T$ bimodule generated by $m$ is finitely generated as a $T$-module and that $M$ is finitely generated as a $\mathrm{g}$ - $T$-bimodule. For example, if we put $T=\mathrm{C}, \mathscr{O}_{T}$ is just the usual BGG-category $\mathscr{O}$.

For $\lambda \in \mathfrak{h}^{*}$ we define the deformed Verma module

$$
\Delta_{T}(\lambda)=U(\mathfrak{g}) \otimes_{U(\mathfrak{b})} T_{\lambda}
$$

where $T_{\lambda}$ denotes the $U(\mathfrak{b})-T$-bimodule $T$ with $\mathfrak{b}$-structure given by the composition $U(\mathfrak{b}) \rightarrow S \stackrel{\lambda+\tau}{\longrightarrow} T$. 
As in [11], we now introduce a functor

$$
d=d_{\tau}: \mathfrak{g} \otimes T-\bmod \longrightarrow \mathfrak{g} \otimes T-\bmod
$$

by letting $d M \subset \operatorname{Hom}_{T}(M, T)^{\sigma}$ be the sum of all deformed weight spaces in the space of homomorphisms of $T$-modules from $M$ to $T$ with its $g$-action twisted by an involutive automorphism $\sigma: \mathfrak{g} \rightarrow \mathfrak{g}$ with $\left.\sigma\right|_{\mathfrak{h}}=-$ id. We now set $\nabla_{T}(\lambda)=d \Delta_{T}(\lambda)$ for $\lambda \in \mathfrak{h}^{*}$ and call it the deformed nabla module. As in [11], one shows $d \nabla_{T}(\lambda) \cong \Delta_{T}(\lambda)$ and that tensoring with a finite dimensional representation $E$ of $g$ commutes with $d$ up to the choice of an isomorphism $d E \cong E$.

Proposition 2.1 ([11], Proposition 2.12.).

(1) For all $\lambda$ the restriction to the deformed weight space of $\lambda$ together with the two canonical identifications $\Delta_{T}(\lambda)_{\lambda} \stackrel{\sim}{\rightarrow} T$ and $\nabla_{T}(\lambda)_{\lambda} \stackrel{\sim}{\rightarrow} T$ induces an isomorphism

$$
\operatorname{Hom}_{\mathscr{O}_{T}}\left(\Delta_{T}(\lambda), \nabla_{T}(\lambda)\right) \stackrel{\sim}{\longrightarrow} T
$$

(2) For $\lambda \neq \mu$ in $\mathfrak{h}^{*}$ we have $\operatorname{Hom}_{\mathscr{O}_{T}}\left(\Delta_{T}(\lambda), \nabla_{T}(\mu)\right)=0$.

(3) For all $\lambda, \mu \in \mathfrak{h}^{*}$ we have $\operatorname{Ext}_{\mathscr{O}_{T}}^{1}\left(\Delta_{T}(\lambda), \nabla_{T}(\mu)\right)=0$.

Corollary 2.2 ([11], Corollary 2.13.). Let $M, N \in \mathscr{O}_{T}$. If $M$ has a $\Delta_{T^{-}}$ flag and $N$ a $\nabla_{T}$-flag, then the space of homomorphisms $\operatorname{Hom}_{\mathscr{O}_{T}}(M, N)$ is a finitely generated free $T$-module and for any homomorphism $T \rightarrow T^{\prime}$ of local deformation algebras the obvious map defines an isomorphism

$$
\operatorname{Hom}_{\mathscr{O}_{T}}(M, N) \otimes_{T} T^{\prime} \stackrel{\sim}{\longrightarrow} \operatorname{Hom}_{\mathscr{O}_{T^{\prime}}}\left(M \otimes_{T} T^{\prime}, N \otimes_{T} T^{\prime}\right)
$$

Proof. This follows from Proposition 2.1 by induction on the length of the $\Delta_{T}$ - and $\nabla_{T}$-flag.

If $\mathfrak{m} \subset T$ is the unique maximal ideal in our local deformation algebra $T$ we set $\mathrm{K}=T / \mathrm{m} T$ for its residue field.

Theorem 2.3 ([5], Propositions 2.1 and 2.6).

(1) The base change $\cdot \otimes_{T} \mathrm{~K}$ gives a bijection

$$
\left\{\begin{array}{c}
\text { simple isomorphism } \\
\text { classes of } \mathscr{O}_{T}
\end{array}\right\} \longleftrightarrow\left\{\begin{array}{c}
\text { simple isomorphism } \\
\text { classes of } \mathscr{O}_{\mathrm{K}}
\end{array}\right\}
$$

(2) The base change $\cdot \otimes_{T} \mathrm{~K}$ gives a bijection

$$
\left\{\begin{array}{c}
\text { projective isomorphism } \\
\text { classes of } \mathscr{O}_{T}
\end{array}\right\} \longleftrightarrow\left\{\begin{array}{c}
\text { projective isomorphism } \\
\text { classes of } \mathscr{O}_{\mathrm{K}}
\end{array}\right\}
$$


REMARK 2.4. The category $\mathscr{O}_{K}$ is the direct summand of the category $\mathscr{O}$ over the Lie algebra $\mathfrak{g} \otimes \mathrm{K}$ consisting of all objects whose weights lie in the complex affine subspace $\tau+\mathfrak{h}^{*}=\tau+\operatorname{Hom}_{\mathrm{C}}(\mathfrak{h}, \mathrm{C}) \subset \operatorname{Hom}_{\mathrm{K}}(\mathfrak{h} \otimes \mathrm{K}, \mathrm{K})$ for $\tau$ being the restriction to $\mathfrak{h}$ of the map that makes K to a $S$-algebra. So the simple objects of $\mathscr{O}_{\mathrm{K}}$ as well as the ones of $\mathscr{O}_{T}$ are parametrized by their highest weight in $\mathfrak{h}^{*}$. Denote by $L_{T}(\lambda)$ the simple object with highest weight $\lambda$. We also use the usual partial order on $\mathfrak{h}^{*}$ to partially order $\tau+\mathfrak{h}^{*}$.

Theorem 2.5 ([5], Propositions 2.4 and 2.7). Let $T$ be a local deformation algebra and $\mathrm{K}$ its residue field. Let $L_{T}(\lambda)$ be a simple object in $\mathcal{O}_{T}$.

(1) There is a projective cover $P_{T}(\lambda)$ of $L_{T}(\lambda)$ in $\mathcal{O}_{T}$. Every projective object in $\mathscr{O}_{T}$ is isomorphic to a direct sum of projective covers.

(2) $P_{T}(\lambda)$ has a Verma flag, i.e., a finite filtration with subquotients isomorphic to Verma modules, and for the multiplicities we have the BGGreciprocity formula

$$
\left(P_{T}(\lambda): \Delta_{T}(\mu)\right)=\left[\Delta_{\mathrm{K}}(\mu): L_{\mathrm{K}}(\lambda)\right]
$$

for all Verma modules $\Delta_{T}(\mu)$ in $\mathscr{O}_{T}$.

(3) Let $T \rightarrow T^{\prime}$ be a homomorphism of local deformation algebras and let $P$ be a projective object in $\mathscr{O}_{T}$. Then $P \otimes_{T} T^{\prime}$ is projective in $\mathscr{O}_{T^{\prime}}$ and the natural transformation

$$
\operatorname{Hom}_{\mathscr{O}_{T}}(P, \cdot) \otimes_{T} T^{\prime} \longrightarrow \operatorname{Hom}_{\mathscr{O}_{T^{\prime}}}\left(P \otimes_{T} T^{\prime}, \cdot \otimes_{T} T^{\prime}\right)
$$

is an isomorphism of functors from $\mathcal{O}_{T}$ to $T^{\prime}$-mod.

REMARK 2.6. Since Fiebig in [5] works over a complex symmetrizable Kac-Moody algebra, he has to introduce truncated subcategories and has to set some finiteness assumptions. In case of a finite dimensional semisimple Lie algebra we do not need these technical tools.

\subsection{Block decomposition}

Let $T$ again denote a local deformation algebra and $\mathrm{K}$ its residue field.

Definition 2.7. Let $\sim_{T}$ be the equivalence relation on $\mathfrak{h}^{*}$ generated by $\lambda \sim_{T} \mu$ if $\left[\Delta_{\mathrm{K}}(\lambda): L_{\mathrm{K}}(\mu)\right] \neq 0$.

Definition 2.8. Let $\Lambda \in \mathfrak{h}^{*} / \sim_{T}$ be an equivalence class. We define $\mathscr{O}_{T, \Lambda}$ to be the full subcategory of $\mathscr{O}_{T}$ consisting of all modules $M$ such that every highest weight of a subquotient of $M$ lies in $\Lambda$. 
Proposition 2.9 ([5], Proposition 2.8). The functor

$$
\begin{gathered}
\bigoplus_{\Lambda \in \mathfrak{h}^{*} / \sim_{T}} \mathcal{O}_{T, \Lambda} \longrightarrow \mathcal{O}_{T} \\
\left(M_{\Lambda}\right)_{\Lambda \in \mathfrak{h}^{*} / \sim_{T}} \longmapsto \bigoplus_{\Lambda \in \mathfrak{h}^{*} / \sim_{T}} M_{\Lambda}
\end{gathered}
$$

is an equivalence of categories.

The isomorphism above is called block decomposition.

Later we will be especially interested in the case $T=R=S_{(0)}$ where $S_{(0)}$ denotes the localization of $S$ at the maximal ideal generated by $\mathfrak{h}$, i.e., the maximal ideal of $0 \in \mathfrak{h}^{*}$. Since $\sim_{R}=\sim_{\mathrm{C}}$, the block decomposition of $\mathscr{O}_{R}$ corresponds to the block decomposition of the BGG-category $\mathcal{O}$ over $g$.

Let $\tau: S \rightarrow$ K be the induced map that makes K into a $S$-algebra. Restricting to $\mathfrak{h}$ and extending with $\mathrm{K}$ yields a $\mathrm{K}$-linear map $\mathfrak{h} \otimes \mathrm{K} \rightarrow \mathrm{K}$ which we will also call $\tau$. Let $\mathscr{R} \supset \mathscr{R}^{+}$be the root system with positive roots according to our data $\mathfrak{g} \supset \mathfrak{b} \supset \mathfrak{h}$. For $\lambda \in \mathfrak{h}_{\mathfrak{K}}^{*}=\operatorname{Hom}_{\mathfrak{K}}(\mathfrak{h} \otimes K, K)$ and $\check{\alpha} \in \mathfrak{h}$ the dual root of a root $\alpha \in \mathscr{R}$ we set $\langle\lambda, \check{\alpha}\rangle_{\mathrm{K}}=\lambda(\check{\alpha}) \in \mathrm{K}$. Let $\mathscr{W}$ be the Weyl group of $(\mathfrak{g}, \mathfrak{h})$.

Definition 2.10. For $\mathscr{R}$ the root system of $\mathfrak{g}$ and $\Lambda \in \mathfrak{h}^{*} / \sim_{T}$ we define

$$
\mathscr{R}_{T}(\Lambda)=\left\{\alpha \in \mathscr{R} \mid\langle\lambda+\tau, \check{\alpha}\rangle_{\mathrm{K}} \in \mathrm{Z} \subset \mathrm{K} \text { for some } \lambda \in \Lambda\right\}
$$

and call it the integral roots corresponding to $\Lambda$. Let $\mathscr{R}_{T}^{+}(\Lambda)$ denote the positive roots in $\mathscr{R}_{T}(\Lambda)$ and set

$$
\mathscr{W}_{T}(\Lambda)=\left\langle\left\{s_{\alpha} \in \mathscr{W} \mid \alpha \in \mathscr{R}_{T}^{+}(\Lambda)\right\}\right\rangle \subset \mathscr{W}
$$

We call it the integral Weyl group with respect to $\Lambda$.

From [5] Corollary 3.3 it follows that

$$
\Lambda=\mathscr{W}_{T}(\Lambda) \cdot \lambda \quad \text { for any } \quad \lambda \in \Lambda
$$

where we denote by the $\rho$-shifted dot-action of the Weyl group, i.e., $w \cdot \rho=$ $w(\lambda+\rho)-\rho$.

Since most of our following constructions commute with base change, we are particularly interested in the case when $T=R_{\mathfrak{p}}$ is a localization of $R$ at a prime ideal $\mathfrak{p}$ of height one. Applying the functor $\cdot \otimes_{R} R_{\mathfrak{p}}$ we split the deformed category $\mathscr{O}_{T}$ into generic and subgeneric blocks which is content of the next

Lemma 2.11 ([6], Lemma 3). Let $\Lambda \in \mathfrak{h}^{*} / \sim_{R}$ and let $\mathfrak{p} \in R$ be a prime ideal. 
(1) If $\check{\alpha} \notin \mathfrak{p}$ for all roots $\alpha \in \mathscr{R}_{R}(\Lambda)$, then $\Lambda$ splits under $\sim_{R_{\mathfrak{p}}}$ into generic equivalence classes.

(2) If $\mathfrak{p}=R \check{\alpha}$ for a root $\alpha \in \mathscr{R}_{R}(\Lambda)$, then $\Lambda$ splits under $\sim_{R_{\mathfrak{p}}}$ into subgeneric equivalence classes of the form $\left\{\lambda, s_{\alpha} \cdot \lambda\right\}$.

We recall that we denote by $P_{T}(\lambda)$ the projective cover of the simple object $L_{T}(\lambda)$. It is indecomposable and up to isomorphism uniquely determined. For an equivalence class $\Lambda \in \mathfrak{h}^{*} / \sim_{T}$ which contains $\lambda$ and is generic, i.e., $\Lambda=\{\lambda\}$, we get $P_{T}(\lambda)=\Delta_{T}(\lambda)$. If $\Lambda=\{\lambda, \mu\}$ and $\mu<\lambda$, we have $P_{T}(\lambda)=\Delta_{T}(\lambda)$ and there is a non-split short exact sequence in $\mathscr{O}_{T}$

$$
0 \longrightarrow \Delta_{T}(\lambda) \longrightarrow P_{T}(\mu) \longrightarrow \Delta_{T}(\mu) \longrightarrow 0
$$

In this case, every endomorphism $f: P_{T}(\mu) \rightarrow P_{T}(\mu)$ maps $\Delta_{T}(\lambda)$ to $\Delta_{T}(\lambda)$ since $\lambda>\mu$. So $f$ induces a commutative diagram

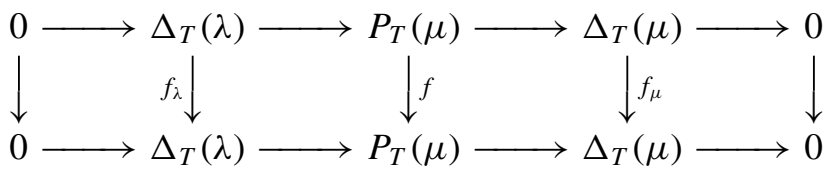

Since endomorphisms of Verma modules correspond to elements of $T$, we get a map

$$
\begin{aligned}
\chi: \operatorname{End}_{\mathcal{O}_{T}}\left(P_{T}(\mu)\right) & \longrightarrow T \oplus T \\
f & \longmapsto\left(f_{\lambda}, f_{\mu}\right)
\end{aligned}
$$

For $\mathfrak{p}=R \check{\alpha}$ we define $R_{\alpha}:=R_{\mathfrak{p}}$ for the localization of $R$ at the prime ideal $\mathfrak{p}$.

Proposition 2.12 ([5], Corollary 3.5). Let $\Lambda \in \mathfrak{h}^{*} / \sim_{R_{\alpha}}$. If $\Lambda=\{\lambda, \mu\}$ and $\lambda=s_{\alpha} \cdot \mu>\mu$, the map $\chi$ from above induces an isomorphism of $R_{\alpha}$-modules

$$
\operatorname{End}_{\mathscr{O}_{R_{\alpha}}}\left(P_{R_{\alpha}}(\mu)\right) \cong\left\{\left(t_{\lambda}, t_{\mu}\right) \in R_{\alpha} \oplus R_{\alpha} \mid t_{\lambda} \equiv t_{\mu} \bmod \check{\alpha}\right\}
$$

\section{Tilting modules and tilting equivalence}

In this chapter, $T$ will be a localization of $R=S_{(0)}$ at a prime ideal $\mathfrak{p} \subset R$ and let $\mathrm{K}$ be its residue field. Let $\lambda \in \mathfrak{h}^{*}$ be such that $\Delta_{K}(\lambda)$ is a simple object in $\mathscr{O}_{\mathrm{K}}$. Thus, we have $\Delta_{\mathrm{K}}(\lambda) \cong \nabla_{\mathrm{K}}(\lambda)$ and the canonical inclusion Can : $\Delta_{T}(\lambda) \hookrightarrow \nabla_{T}(\lambda)$ becomes an isomorphism after applying $\cdot \otimes_{T} \mathrm{~K}$. So by Nakayama's lemma, we conclude that Can was bijective already. 


\subsection{Deformed tilting modules}

Definition 3.1. By $\mathscr{K}_{T}$ we denote the full subcategory of $\mathscr{O}_{T}$ which

(1) includes the self-dual deformed Verma modules

(2) is stable under tensoring with finite dimensional $g$-modules

(3) is stable under forming direct sums and summands.

Remark 3.2. For $T=S / S \mathfrak{h}=\mathrm{C}$ the category $\mathscr{K}_{T}$ is just the usual subcategory of tilting modules of the category $\mathscr{O}$ over $g$. In general, $\mathscr{K}_{\mathrm{K}}$ is the category of tilting modules of category $\mathcal{O}$ over the Lie algebra $\mathfrak{g} \otimes \mathrm{K}$ whose weights live in the affine complex subspace $\tau+\mathfrak{h}^{*} \subset \operatorname{Hom}_{\mathrm{K}}(\mathfrak{h} \otimes \mathrm{K}, \mathrm{K})$, where $\tau: \mathfrak{h} \otimes \mathrm{K} \rightarrow \mathrm{K}$ comes from the map that makes $\mathrm{K}$ into an $S$-algebra.

Proposition 3.3. The base change $\cdot \otimes_{T} \mathrm{~K}$ gives a bijection

$$
\left\{\begin{array}{c}
\text { isomorphism classes } \\
\text { of } \mathscr{K}_{T}
\end{array}\right\} \longleftrightarrow\left\{\begin{array}{c}
\text { isomorphism classes } \\
\text { of } \mathscr{K}_{\mathrm{K}}
\end{array}\right\}
$$

Proof. For $K, H \in \mathscr{K}_{T}$ with $K \otimes_{T} \mathrm{~K} \cong H \otimes_{T} \mathrm{~K}$ we conclude $K \cong H$ from Nakayama's lemma applied to the weight spaces, since the weight spaces of tilting modules are finitely generated and free over $T$. This shows injectivity.

For surjectivity we only have to show that every indecomposable tilting module in $\mathscr{O}_{\mathrm{K}}$ has an indecomposable preimage in $\mathscr{K}_{T}$. Since we are not working over a complete local ring we cannot apply the idempotent lifting lemma as in the proof of Proposition 3.4. in [11]. Rather, the proof works very similar to the proof of Theorem 6 in [8].

Let $K \in \mathscr{O}_{\mathrm{K}}$ be an indecomposable tilting module. For the sake of simplicity, we will assume the highest weight of $K$ to be regular. The singular case is treated analogously.

If the highest weight $\lambda$ of $K$ is minimal in its equivalence class under $\sim_{T}$ then, $K \cong \Delta_{K}(\lambda) \cong \nabla_{K}(\lambda)$ and we can take $\Delta_{T}(\lambda)$ as a preimage of $K$ in $\mathscr{K}_{T}$.

Now denote by $\bar{\lambda}$ the equivalence class of $\lambda$ in $\mathfrak{h}^{*} / \sim_{T}$ and let $\lambda=w \cdot \mu$ with $w \in \mathscr{W}_{T}(\bar{\lambda})$ and $\mu$ minimal in $\mathscr{W}_{T}(\bar{\lambda}) \cdot \lambda$. In addition, let $w=s_{1} s_{2} \ldots s_{n}$ be a minimal expression of $w$ with simple reflections $s_{i} \in \mathscr{W}_{T}(\bar{\lambda})$. We denote by $\theta_{i}=\theta_{i}^{\mathrm{K}}$ the translation functor of $\mathscr{O}_{\mathrm{K}}$ through the $s_{i}$-wall. Then $K$ is a direct summand of the tilting module

$$
M=\theta_{1} \ldots \theta_{n} \Delta_{\mathrm{K}}(\mu)
$$

and we get a decomposition $M \cong K \oplus K^{\prime} . K^{\prime}$ decomposes into indecomposable tilting modules with highest weights of the form $w^{\prime} \cdot \mu$ and $l(w)>l\left(w^{\prime}\right)$ where $l$ denotes the length of a Weyl group element. By using induction on 
the length of $w$, we get a preimage $\tilde{K} \in \mathscr{K}_{T}$ of $K^{\prime}$ together with a splitting inclusion

$$
\tilde{K} \otimes_{T} \mathrm{~K} \hookrightarrow M
$$

Using Nakayama's lemma, this induces a splitting lift

$$
\tilde{K} \hookrightarrow \theta_{1}^{T} \ldots \theta_{n}^{T} \Delta_{T}(\mu)
$$

where $\theta_{i}^{T}$ denotes the $T$-deformed wall-crossing functor of $\mathscr{O}_{T}$ corresponding to $\theta_{i}^{K}$. Finally, the cokernel of this inclusion is the indecomposable tilting module in $\mathscr{K}_{T}$ we were looking for.

\subsection{Tilting functor}

Let $\mathcal{M}_{T}$ denote the subcategory of all modules in $\mathscr{O}_{T}$ admitting a Verma flag. We recall the map $\tau: S \rightarrow T$ which makes $T$ into a $S$-algebra. We get a new $S$-algebra structure on $T$ via $\tau \circ \gamma: S \rightarrow T$, where $\gamma: S \rightarrow S$ is the isomorphism given by $\gamma(h)=-h$ for all $h \in \mathfrak{h}$. We denote this new $S$-algebra by $\bar{T}$. Let $S_{2 \rho}$ be the semiregular $U(\mathrm{~g})$-bimodule of [10]. If $N$ is a $\mathrm{g} \otimes T$-module which decomposes into weight spaces we get a $\bar{T}$-module

$$
N^{\star}=\bigoplus_{\lambda \in h^{*}} \operatorname{Hom}_{T}\left(N_{\lambda}, T\right)
$$

Then we get a $\mathfrak{g} \otimes \bar{T}$-module via the $\mathfrak{g}$-action $(X f)(v)=-f(X v)$ for all $X \in \mathfrak{g}, f \in N^{\star}$ and $v \in N$.

Now we get a functor

$$
t_{T}^{\prime}: \mathscr{M}_{T} \longrightarrow \mathcal{M}_{\bar{T}}^{o p p}
$$

by setting $t_{T}^{\prime}(M)=\left(S_{2 \rho} \otimes_{U(\mathfrak{g})} M\right)^{\star}$.

For $T$ a localization of $S$ at a prime ideal $\mathfrak{p}$ which is stable under $\gamma$, for a residue field of this or for the ring $\mathrm{C} \llbracket v \rrbracket$ of formal power series coming from a line $C \lambda \subset \mathfrak{h}^{*}, \gamma$ induces an isomorphism of $S$-algebras $\gamma: T \stackrel{\sim}{\longrightarrow} \bar{T}$ which induces an equivalence of categories

$$
\gamma: \mathscr{M}_{T} \longrightarrow \mathscr{M}_{\bar{T}}
$$

Theorem 3.4 ([6], Section 2.6). The functor $t_{T}=\left(S_{2 \rho} \otimes_{U(\mathfrak{g})} \cdot\right)^{\star} \circ \gamma$ induces an equivalence of categories

$$
t_{T}: \mathscr{M}_{T} \longrightarrow \mathcal{M}_{T}^{o p p}
$$

which makes short exact sequences to short exact sequences and sends a Verma module $\Delta_{T}(\lambda)$ to the Verma module $\Delta(-2 \rho-\lambda)$ for any weight $\lambda \in \mathfrak{h}^{*}$. 
REMARK 3.5. The non-deformed tilting functor for category $\mathscr{O}$ over a Zgraded Lie algebra was discovered by Soergel in [10]. This work was mainly motivated by the article [1] of Arkhipov in which he proves a semi-infinite duality between certain categories over a Z-graded associative algebra. In [6] Fiebig formulates a deformed version of the tilting functor which is the one we use in the above theorem.

Proposition 3.6. Let $\lambda \in \mathfrak{h}^{*}$. Then

$$
t_{T}\left(P_{T}(\lambda)\right) \cong K_{T}(-2 \rho-\lambda)
$$

where $P_{T}(\lambda)$ denotes the indecomposable projective cover of $L_{T}(\lambda)$ and $K_{T}(\mu)$ the up to isomorphism unique indecomposable deformed tilting module with highest weight $\mu \in \mathfrak{h}^{*}$.

Proof. We set $\mu=-2 \rho-\lambda$. This proof is very similar to the proof of Proposition 3.1 of [9]. As we have already seen, the tilting module $K_{T}(\mu)$ can be described as the up to isomorphism unique indecomposable module in $\mathscr{O}_{T}$ with the properties:

(1) $K_{T}(\mu)$ admits a Verma flag

(2) $K_{T}(\mu)$ has a $\nabla_{T}$-flag

(3) $K_{T}(\mu)_{\mu}$ is free of rank one over $T$

(4) If $\gamma$ is a weight of $K_{T}(\mu)$, we have $\gamma \leq \mu$.

Since $t_{T}$ is fully faithful, we already conclude the indecomposability of $t_{T}\left(P_{T}(\lambda)\right)$. Theorem 3.4 also tells us that $t_{T}\left(P_{T}(\lambda)\right)$ has a Verma flag. By BGG-reciprocity we get a short exact sequence

$$
N \hookrightarrow P_{T}(\lambda) \rightarrow \Delta_{T}(\lambda)
$$

where $N$ has a Verma flag in which the occurring weights are strictly larger than $\lambda$. By applying $t_{T}$ we get a new short exact sequence

$$
t_{T}(N) \ll t_{T}\left(P_{T}(\lambda)\right) \hookleftarrow t_{T}\left(\Delta_{T}(\lambda)\right)
$$

By induction on the length of the Verma flag of $N$ we conclude that the weights of $t_{T}(N)$ are strictly smaller than $\mu$. So the weight space $\left(t_{T}\left(P_{T}(\lambda)\right)\right)_{\mu}$ is free of rank one, since $t_{T}\left(\Delta_{T}(\lambda)\right) \cong \Delta_{T}(\mu)$. Now, $P_{T}(\lambda)$ being projective and $t_{T}$ being fully faithful, we get

$$
\operatorname{Ext}_{\mathscr{O}_{T}}^{1}\left(\Delta_{T}(\delta), t_{T}\left(P_{T}(\lambda)\right)\right)=0 \quad \forall \delta \in \mathfrak{h}^{*}
$$


Now we set $D_{T}=t_{T}\left(P_{T}(\lambda)\right)$ and consider the diagramm

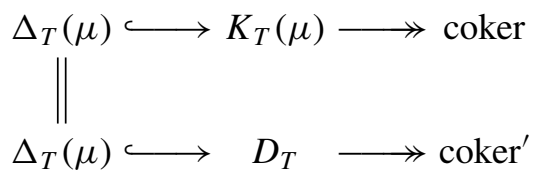

Since coker has a Verma flag we conclude $\operatorname{Ext}_{\mathscr{O}_{T}}^{1}\left(\right.$ coker, $\left.D_{T}\right)=0$ using induction on the length of a Verma flag of coker. So the restriction induces a surjection $\operatorname{Hom}_{\mathscr{O}_{T}}\left(K_{T}(\mu), D_{T}\right) \rightarrow \operatorname{Hom}_{\mathscr{O}_{T}}\left(\Delta_{T}(\mu), D_{T}\right)$ and we get a map $\alpha: K_{T}(\mu) \rightarrow D_{T}$ which induces the identity on $\Delta_{T}(\mu)$. For the same reason we also get a map $\beta: D_{T} \rightarrow K_{T}(\mu)$ with the same property, since coker' has a Verma flag while $K_{T}(\mu)$ admits a nabla flag which implies $\operatorname{Ext}_{\mathscr{O}_{T}}^{1}\left(\right.$ coker' $\left.^{\prime}, K_{T}(\mu)\right)=0$ by Proposition 2.1. Applying the base change functor $\cdot \otimes_{T} \mathrm{~K}$, we get two maps $\varphi:=(\beta \circ \alpha) \otimes \mathrm{id}_{\mathrm{K}}: K_{\mathrm{K}}(\mu) \rightarrow K_{\mathrm{K}}(\mu)$ and $\psi:=(\alpha \circ \beta) \otimes \operatorname{id}_{\mathrm{K}}: D_{T} \otimes_{T} \mathrm{~K} \rightarrow D_{T} \otimes_{T} \mathrm{~K}$ which induce the identity on $\Delta_{\mathrm{K}}(\mu)$. We conclude that $\varphi$ and $\psi$ are not nilpotent and since $D_{T} \otimes_{T} \mathrm{~K}$ and $K_{\mathrm{K}}(\mu)$ have finite length and are indecomposable, it follows from the Fitting lemma that $\varphi$ and $\psi$ are isomorphisms. Now by Nakayama's lemma applied to all weight spaces, we conclude $K_{T}(\mu) \cong D_{T}$.

Lemma 3.7. Let $T \rightarrow T^{\prime}$ be a homomorphism of $S$-algebras where also $T^{\prime}$ is a localization of $S$ at a prime ideal which is stable under $\gamma$, its residue field or the ring of formal power series $\mathrm{C} \llbracket v \rrbracket$. Let $M, N \in \mathcal{M}_{T}$ and let $M$ be projective in $\mathcal{O}_{T}$. Then the diagram

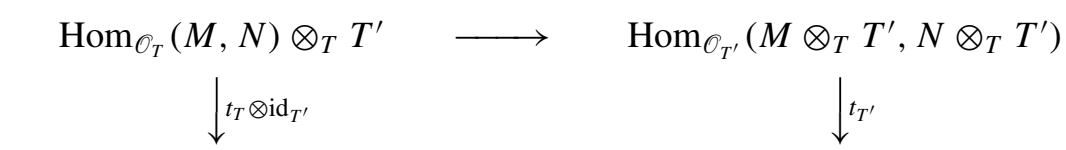

$\operatorname{Hom}_{\mathscr{O}_{T}}\left(t_{T}(N), t_{T}(M)\right) \otimes_{T} T^{\prime} \longrightarrow \operatorname{Hom}_{\mathscr{C}_{T^{\prime}}}\left(t_{T^{\prime}}\left(N \otimes_{T} T^{\prime}\right), t_{T^{\prime}}\left(M \otimes_{T} T^{\prime}\right)\right)$

commutes, where the horizontals are the base change isomorphisms and the verticals are induced by the tilting functors $t_{T}$ resp. $t_{T^{\prime}}$.

Proof. All composition factors of the tilting functor commute with base change in the sense of the lemma.

\section{The Jantzen and Andersen filtrations}

We fix a deformed tilting module $K \in \mathscr{K}_{T}$ and let $\lambda \in \mathfrak{h}^{*}$. The composition of homomorphisms induces a $T$-bilinear pairing

$$
\begin{aligned}
\operatorname{Hom}_{O_{T}}\left(\Delta_{T}(\lambda), K\right) \times \operatorname{Hom}_{\mathcal{O}_{T}}\left(K, \nabla_{T}(\lambda)\right) & \longrightarrow \operatorname{Hom}_{O_{T}}\left(\Delta_{T}(\lambda), \nabla_{T}(\lambda)\right) \cong T \\
(\varphi, \psi) & \longmapsto \psi \varphi \varphi
\end{aligned}
$$


For any $T$-module $H$ we denote by $H^{*}$ the $T$-module $\operatorname{Hom}_{T}(H, T)$. As in [11] Section 4 one shows that for $T$ a localization of $S$ at a prime ideal $\mathfrak{p}$ or for $T=\mathrm{C} \llbracket v \rrbracket$ our pairing is nondegenerate and induces an injective map

$$
E=E_{T}^{\lambda}(K): \operatorname{Hom}_{\mathscr{O}_{T}}\left(\Delta_{T}(\lambda), K\right) \longrightarrow\left(\operatorname{Hom}_{\mathscr{O}_{T}}\left(K, \nabla_{T}(\lambda)\right)\right)^{*}
$$

of finitely generated free $T$-modules.

Let $\mathrm{C} \delta \subset \mathfrak{h}^{*}$ be a line not contained in any hyperplane corresponding to a reflection of the Weyl group. We set $T=\mathrm{C} \llbracket v \rrbracket$ to be the ring of formal power series around the origin in $C \delta$. Now we get a filtration on $\operatorname{Hom}_{\mathscr{O}_{T}}\left(\Delta_{T}(\lambda), K\right)$ by taking the preimages of $\left(\operatorname{Hom}_{\mathcal{O}_{T}}\left(K, \nabla_{T}(\lambda)\right)\right)^{*} \cdot v^{i}$ for $i=0,1,2, \ldots$ under E.

Definition 4.1 ([11], Definition 4.2). Given $K_{\mathrm{C}} \in \mathscr{K}_{\mathrm{C}}$ a tilting module of $\mathcal{O}$ and $K \in \mathscr{K}_{\complement \llbracket v \rrbracket}$ a preimage of $K_{\mathrm{C}}$ under the functor $\cdot \otimes_{\complement \llbracket v \rrbracket} \mathrm{C}$, which is possible by Proposition 3.3 with $S \rightarrow \mathrm{C} \llbracket v \rrbracket$ the restriction to a formal neighbourhood of the origin in the line $\mathrm{C} \rho$, then the image of the filtration defined above under specialization $\cdot \otimes_{\complement \llbracket v \rrbracket} \mathrm{C}$ is called the Andersen filtration on $\operatorname{Hom}_{\mathfrak{g}}\left(\Delta(\lambda), K_{\mathrm{C}}\right)$.

In a similar manner we get the Jantzen filtration on a Verma module:

Definition 4.2. Let $\varphi: \Delta_{\complement \llbracket v \rrbracket}(\lambda) \hookrightarrow \nabla_{\complement \llbracket v \rrbracket}(\lambda)$ be a generator of the $C \llbracket v \rrbracket-$ module $\operatorname{Hom}_{\mathcal{C}_{\complement \llbracket v \|}}\left(\Delta_{\complement \llbracket v \rrbracket}(\lambda), \nabla_{\complement \llbracket v \rrbracket}(\lambda)\right)$. Then $\Delta_{C \llbracket v \|}(\lambda)$ yields a filtration by the preimages of $v^{i} \cdot \nabla_{\complement \llbracket v \|}(\lambda)(i=0,1,2, \ldots)$ under $\varphi$. The induced filtration on $\Delta(\lambda)$ after specialization is called Jantzen filtration.

The Jantzen filtration on a Verma module $\Delta(\lambda)$ induces a filtration on the vector space $\operatorname{Hom}_{\mathfrak{g}}(P, \Delta(\lambda))$, where $P$ is a projective object in $\mathcal{O}$. Now consider again the embedding $\Delta_{\complement \llbracket v \rrbracket}(\lambda) \hookrightarrow \nabla_{\complement \llbracket v \rrbracket}(\lambda)$. Let $P_{\complement \llbracket v \|}$ denote the up to isomorphism unique projective object in $\mathscr{O}_{\complement \llbracket v \rrbracket}$ that maps to $P$ under $\cdot \otimes_{\complement \llbracket v \rrbracket} \mathrm{C}$, which is possible by Theorem 2.3. Then we get the same filtration by taking the preimages of $\operatorname{Hom}_{\mathcal{O}_{\complement \llbracket v \|}}\left(P_{\complement \llbracket v \|}, \nabla_{\complement \llbracket v \rrbracket}(\lambda)\right) \cdot v^{i}, i=0,1,2, \ldots$, under the induced inclusion

$$
J=J_{T}^{\lambda}(P): \operatorname{Hom}_{\mathscr{O}_{T}}\left(P_{T}, \Delta_{T}(\lambda)\right) \longrightarrow \operatorname{Hom}_{\mathscr{O}_{T}}\left(P_{T}, \nabla_{T}(\lambda)\right)
$$

for $T=\mathrm{C} \llbracket v \rrbracket$ and taking the images of these filtration layers under the map $\operatorname{Hom}_{\mathscr{O}_{T}}\left(P_{T}, \Delta_{T}(\lambda)\right) \rightarrow \operatorname{Hom}_{\mathrm{g}}(P, \Delta(\lambda))$ induced by $\cdot \otimes_{T} \mathrm{C}$.

For what follows, we define $\mu^{\prime}=-2 \rho-\mu$ and $\lambda^{\prime}=-2 \rho-\lambda$. To avoid ambiguity, we sometimes write $(\cdot)^{*_{T}}$, when we mean the $T$-dual of the $T$ module in brackets. 
Theorem 4.3. Let $\lambda, \mu \in \mathfrak{h}^{*}$. Denote by $R=S_{(0)}$ the localization of $S$ at 0 . There exists an isomorphism $L=L_{R}(\lambda, \mu)$ which makes the diagram

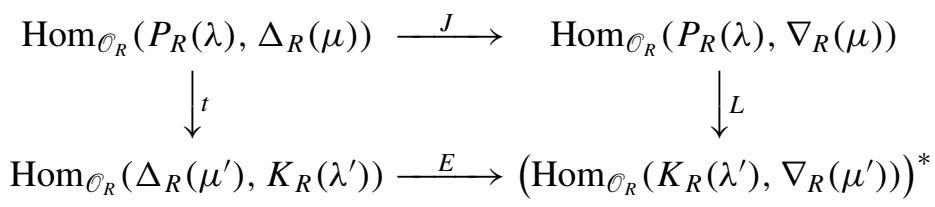

commutative. Here $J=J_{R}^{\mu}\left(P_{R}(\lambda)\right)$ and $E=E_{R}^{\mu^{\prime}}\left(K_{R}\left(\lambda^{\prime}\right)\right)$ denote the inclusions defined above and $t=t_{R}$ denotes the isomorphism induced by the tilting functor.

Proof. If $\lambda$ is not contained in the equivalence class of $\mu$ under $\sim_{R}=\sim_{C}$ all Hom-spaces occurring in the diagram are 0 by block decomposition and the assertion of the proposition is true.

So let us assume $\lambda$ to be in the equivalence class of $\mu$. It is easy to see that $J$ and $E$ commute with base change and we have also verified this property for $t$ in Lemma 3.7 already. Let $\mathfrak{p} \subset R$ be a prime ideal of height 1 . We abbreviate Hom $=\operatorname{Hom}_{\mathscr{O}_{R_{\mathrm{p}}}}, P=P_{R}(\lambda), K=K_{R}\left(\lambda^{\prime}\right), \Delta=\Delta_{R_{\mathrm{p}}}(\mu), \nabla=$ $\nabla_{R_{\mathfrak{p}}}(\mu), \Delta^{\prime}=\Delta_{R_{\mathrm{p}}}\left(\mu^{\prime}\right)$ and $\nabla^{\prime}=\nabla_{R_{\mathrm{p}}}\left(\mu^{\prime}\right)$. After applying $\cdot \otimes_{R} R_{\mathfrak{p}}$ to our diagram and the base change isomorphisms of Theorem 2.5 and Corollary 2.2 we get a diagram of $R_{\mathfrak{p}}$-modules

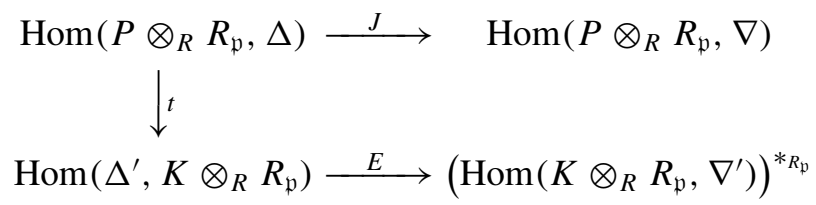

where we omit the index $R_{\mathfrak{p}}$ for $t, J$ and $E$.

We want to show that we get an isomorphism $L_{R_{\mathfrak{p}}}$ of $R_{\mathfrak{p}}$-modules as the missing right vertical of the upper diagram to make it commutative for every prime ideal $\mathfrak{p} \subset R$ of height 1 . By block decomposition we get

$$
P_{R}(\lambda) \otimes_{R} R_{\mathfrak{p}} \cong \bigoplus_{i=1}^{n} P_{R_{\mathfrak{p}}}\left(\lambda_{i}\right)
$$

for certain indecomposable projective objects $P_{R_{\mathrm{p}}}\left(\lambda_{i}\right) \in \mathscr{O}_{R_{\mathrm{p}}}$ and $\lambda_{i} \in \bar{\lambda}$ where $\bar{\lambda}$ denotes the equivalence class of $\lambda$ under $\sim_{R}=\sim_{\mathrm{C}}$. Since $t$ is fully faithful and respects base change we also get a decomposition

$$
K_{R}\left(\lambda^{\prime}\right) \otimes_{R} R_{\mathfrak{p}} \cong t_{R_{\mathfrak{p}}}\left(P_{R}(\lambda) \otimes_{R} R_{\mathfrak{p}}\right) \cong \bigoplus_{i=1}^{n} t_{R_{\mathfrak{p}}}\left(P_{R_{\mathfrak{p}}}\left(\lambda_{i}\right)\right)
$$


It is easy to see that $J, E$ and $t$ respect these decompositions. Hence, we get in formulas

$$
\begin{aligned}
J\left(\operatorname{Hom}_{\mathscr{O}_{R_{p}}}\left(P_{R_{p}}\left(\lambda_{i}\right), \Delta_{R_{p}}(\mu)\right)\right) & \subset \operatorname{Hom}_{\mathscr{O}_{R_{p}}}\left(P_{R_{p}}\left(\lambda_{i}\right), \nabla_{R_{p}}(\mu)\right) \\
t\left(\operatorname{Hom}_{\mathscr{O}_{R_{p}}}\left(P_{R_{p}}\left(\lambda_{i}\right), \Delta_{R_{p}}(\mu)\right)\right) & =\operatorname{Hom}_{O_{R_{p}}}\left(t\left(\Delta_{R_{p}}(\mu)\right), t\left(P_{R_{p}}\left(\lambda_{i}\right)\right)\right) \\
E\left(\operatorname{Hom}_{O_{R_{p}}}\left(t\left(\Delta_{R_{p}}(\mu)\right), t\left(P_{R_{p}}\left(\lambda_{i}\right)\right)\right)\right) & \subset\left(\operatorname{Hom}_{\mathscr{O}_{R_{p}}}\left(t\left(P_{R_{p}}\left(\lambda_{i}\right)\right), \nabla_{R_{p}}\left(\mu^{\prime}\right)\right)\right)^{* R_{p}}
\end{aligned}
$$

where we omit the index $R_{\mathfrak{p}}$ of our maps.

Let $Q=\operatorname{Quot}(S)$ be the quotient field of $S$. Since all deformed Verma modules over $Q$ are simple, we get $\Delta_{Q}(\mu) \cong \nabla_{Q}(\mu)$ and $\Delta_{Q}\left(\mu^{\prime}\right) \cong \nabla_{Q}\left(\mu^{\prime}\right)$, respectively. Hence, after applying $\cdot \otimes_{R} Q$ to our diagram we get an isomorphism $L_{Q}$ which makes the following diagram commutative

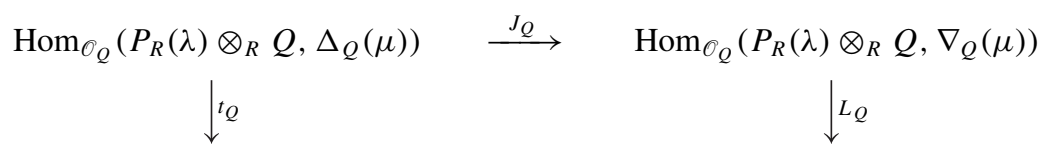

$\operatorname{Hom}_{\mathscr{O}_{Q}}\left(t_{Q}\left(\Delta_{Q}(\mu)\right), t_{Q}\left(P_{R}(\lambda) \otimes_{R} Q\right)\right) \stackrel{E_{Q}}{\longrightarrow}\left(\operatorname{Hom}_{\mathscr{Q}_{Q}}\left(t_{Q}\left(P_{R}(\lambda) \otimes_{R} Q\right), \nabla_{Q}\left(\mu^{\prime}\right)\right)\right)^{* Q}$

Let $\mathfrak{p} \subset R$ be a prime ideal of height one. So $\mathfrak{p}=R \gamma$ for an irreducible element $\gamma \in R$. If $\gamma \notin C \check{\alpha}$ for all $\alpha \in \mathscr{R}^{+}$or $\gamma \in \mathrm{C} \check{\alpha}$ for one $\alpha \in \mathscr{R}$ but with $\langle\mu+\rho, \check{\alpha}\rangle_{\mathrm{C}} \notin \mathrm{Z}$, the block of $\mu$ in $\mathscr{O}_{R_{\mathrm{p}}}$ is generic by Lemma 2.11 and we conclude $P_{R_{\mathrm{p}}}(\mu) \cong \Delta_{R_{\mathrm{p}}}(\mu) \cong \nabla_{R_{\mathrm{p}}}(\mu)$. But in this case all maps $J_{R_{\mathrm{p}}}, E_{R_{\mathrm{p}}}$ and $t_{R_{\mathfrak{p}}}$ are isomorphisms and so we get the claimed $R_{\mathfrak{p}}$-isomorphism $L_{R_{\mathfrak{p}}}$ of our diagram (2). So we can assume $\mathfrak{p}=R \check{\alpha}$ for some $\alpha \in \mathscr{R}^{+}$and $\langle\mu+\rho, \check{\alpha}\rangle_{\mathrm{C}} \in \mathbf{Z}$.

By Proposition 4.5 below we get

$$
\begin{aligned}
L_{Q}\left(\operatorname{Hom}_{\mathscr{O}_{R_{\mathfrak{p}}}}\right. & \left.\left(P_{R}(\lambda) \otimes_{R} R_{\mathfrak{p}}, \nabla_{R_{\mathfrak{p}}}(\mu)\right)\right) \\
& =\left(\operatorname{Hom}_{\mathscr{O}_{R_{\mathfrak{p}}}}\left(t\left(P_{R}(\lambda)\right) \otimes_{R} R_{\mathfrak{p}}, \nabla_{R_{\mathfrak{p}}}\left(\mu^{\prime}\right)\right)\right)^{*_{R_{\mathfrak{p}}}} \\
& \subset\left(\operatorname{Hom}_{\mathscr{O}_{Q}}\left(t\left(P_{R}(\lambda)\right) \otimes_{R} Q, \nabla_{Q}\left(\mu^{\prime}\right)\right)\right)^{* Q}
\end{aligned}
$$

Here we identify both $R_{\mathfrak{p}}$-modules with submodules of the according $Q$-vector spaces.

In conclusion, for every prime ideal $\mathfrak{p} \subset R$ of height 1 the restriction of $L_{Q}$ to the according $R_{\mathfrak{p}}$-lattice leads to the sought-after $R_{\mathfrak{p}}$-isomorphism $L_{R_{\mathfrak{p}}}$ that makes the diagram (2) commutative. Since $E, J$ and $t$ respect base change we get an isomorphism which we will also call $L=L_{R_{\mathrm{p}}}$ that makes

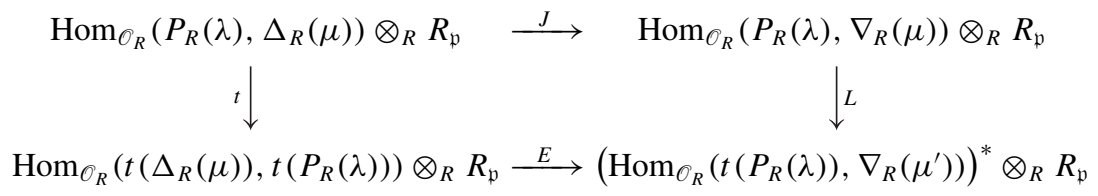
commutative. 
But then we also get an isomorphism $L_{R}$ which is the restriction of the $Q$-linear map $L_{Q}$ to $\operatorname{Hom}_{\mathscr{O}_{R}}\left(P_{R}(\lambda), \nabla_{R}(\mu)\right)$ and makes the diagram

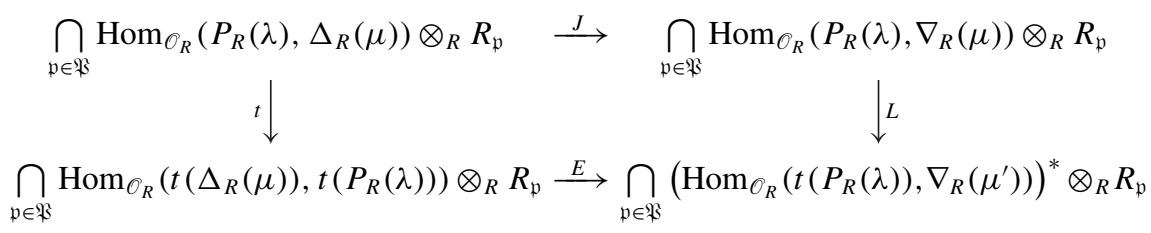

commutative, where $\mathfrak{R}$ denotes the set of all prime ideals of $R$ of height one. Since all Hom-spaces of this diagram are finitely generated free $R$-modules, it equals diagram (1) which finishes the proof.

Corollary 4.4. Let $P \in \mathcal{O}$ be a projective object, $\mu \in \mathfrak{h}^{*}$ and let $t=t_{\mathrm{c}}$ be the tilting functor. Then $K=t(P)$ is a tilting object in $\mathcal{O}$ and the isomorphism $t: \operatorname{Hom}_{\mathfrak{g}}(P, \Delta(\mu)) \stackrel{\sim}{\rightarrow} \operatorname{Hom}_{\mathfrak{g}}(\Delta(-2 \rho-\mu), K)$ induced by the tilting functor identifies the filtration induced by the Jantzen filtration with the Andersen filtration.

Proof. Consider the restriction map $S=\mathrm{C}\left[\mathfrak{h}^{*}\right] \rightarrow \mathrm{C} \llbracket v \rrbracket$ to a formal neighbourhood of the origin in the line $\mathrm{C} \rho$. Since this map factors through $R$, we get a homomorphism $R \rightarrow \mathrm{C} \llbracket v \rrbracket$ of $S$-algebras. Since the maps from the Theorem above commute with base change $\cdot \otimes_{R} C \llbracket v \rrbracket$, we get a commuting diagram

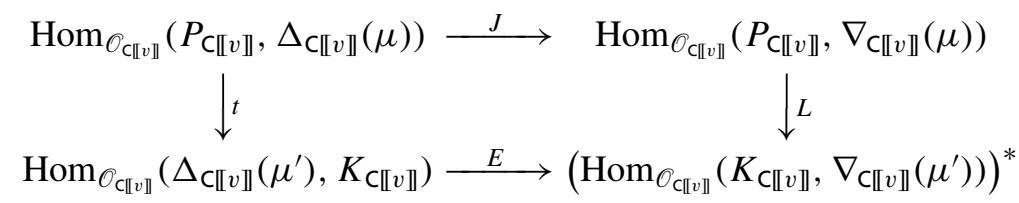

where $P_{\complement \llbracket v \|}\left(\right.$ resp. $\left.K_{\complement \llbracket v \|}\right)$ denotes the unique (up to isomorphism) deformed projective (resp. tilting) module in $\mathscr{O}_{\mathrm{C} \llbracket v \rrbracket}$ that maps to $P$ (resp. $K$ ) under $\cdot \otimes_{\complement \llbracket v \rrbracket}$

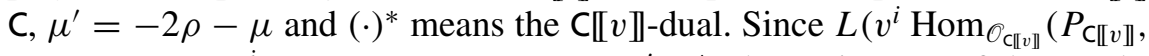
$\left.\left.\nabla_{\complement \llbracket v \rrbracket}(\mu)\right)\right)=v^{i}\left(\operatorname{Hom}_{\mathcal{O}_{\complement \llbracket v \rrbracket}}\left(K_{\complement \llbracket v \rrbracket}, \nabla_{\complement \llbracket v \|}\left(\mu^{\prime}\right)\right)\right)^{*}$, the preimages of these submodules under $J$ resp. $E$ are identified by $t$. Applying $\cdot \otimes_{\complement \complement}\|v\| C$ to the left vertical of the diagram yields the claim.

For the next Proposition we assume $\mathfrak{p}=R \check{\alpha}$ for some $\alpha \in \mathscr{R}^{+}$and $\langle\mu+$ $\rho, \check{\alpha}\rangle_{\mathrm{C}} \in \mathbf{Z}$.

Proposition 4.5. With the notation and identifications from the proof of Theorem 4.3 we get

$$
\begin{aligned}
L_{Q}\left(\operatorname{Hom}_{\mathscr{O}_{R_{\mathrm{p}}}}\left(P_{R}(\lambda) \otimes_{R} R_{\mathfrak{p}}, \nabla_{R_{\mathfrak{p}}}(\mu)\right)\right) \\
=\left(\operatorname{Hom}_{\mathscr{O}_{R_{\mathfrak{p}}}}\left(t\left(P_{R}(\lambda)\right) \otimes_{R} R_{\mathfrak{p}}, \nabla_{R_{\mathrm{p}}}\left(\mu^{\prime}\right)\right)\right)^{*_{R_{\mathfrak{p}}}} \\
\quad \subset\left(\operatorname{Hom}_{\mathscr{O}_{Q}}\left(t\left(P_{R}(\lambda)\right) \otimes_{R} Q, \nabla_{Q}\left(\mu^{\prime}\right)\right)\right)^{*_{Q}}
\end{aligned}
$$


Proof. Since all maps $t, J, E$ respect the decomposition of $P_{R}(\lambda) \otimes_{R} R_{\mathfrak{p}} \cong$ $\bigoplus_{i=1}^{n} P_{R_{\mathrm{p}}}\left(\lambda_{i}\right)$, we only have to prove the statement for the indecomposable summands of this decomposition. In formulas we want to show

$$
L_{Q}\left(\operatorname{Hom}_{\mathcal{O}_{R_{\mathrm{p}}}}\left(P_{R_{\mathrm{p}}}\left(\lambda_{i}\right), \nabla_{R_{\mathrm{p}}}(\mu)\right)\right)=\left(\operatorname{Hom}_{\mathcal{O}_{R_{\mathrm{p}}}}\left(t\left(P_{R_{\mathrm{p}}}\left(\lambda_{i}\right)\right), \nabla_{R_{\mathrm{p}}}\left(\mu^{\prime}\right)\right)\right)^{*_{R_{\mathrm{p}}}}
$$

for all $i \in\{1, \ldots, n\}$. Here, the right side is again meant to be the $R_{\mathfrak{p}}$-lattice in the $Q$-vector space $\operatorname{Hom}_{\mathcal{O}_{Q}}\left(t\left(P_{R_{\mathrm{p}}}\left(\lambda_{i}\right)\right) \otimes_{R_{\mathrm{p}}} Q, \nabla_{Q}\left(\mu^{\prime}\right)\right)^{*}$.

Both Hom-spaces in (3) are free and of the same rank over $R_{\mathfrak{p}}$. From the description of the projective covers in the generic and subgeneric case it follows

$$
\begin{aligned}
\operatorname{rk}_{R_{\mathrm{p}}}\left(\operatorname{Hom}_{\mathscr{O}_{R_{\mathrm{p}}}}\left(P_{R_{\mathrm{p}}}\left(\lambda_{i}\right), \nabla_{R_{\mathrm{p}}}(\mu)\right)\right) & =\operatorname{dim}_{\mathrm{K}}\left(\operatorname{Hom}_{\mathscr{O}_{\mathrm{K}}}\left(P_{\mathrm{K}}\left(\lambda_{i}\right), \nabla_{\mathrm{K}}(\mu)\right)\right) \\
& =\left(P_{\mathrm{K}}\left(\lambda_{i}\right): \Delta_{\mathrm{K}}(\mu)\right) \leq 1
\end{aligned}
$$

where $\mathrm{K}$ denotes the residue field of $R_{\mathfrak{p}}$.

Now we will proceed in several steps to prove (3). From the choice of $\mathfrak{p}$ it follows that the equivalence class of $\mu$ under $\sim_{R_{p}}$ equals $\left\{\mu, s_{\alpha} \cdot \mu\right\}$ where again $s_{\alpha}$ is the reflection corresponding to the root $\alpha \in \mathscr{R}$ acting by the dot-action on $\mathfrak{h}^{*}$. If $\mu=s_{\alpha} \cdot \mu$, we are in the generic case and our maps are isomorphisms which proves the claim for this case. So let us further assume $\mu \neq s_{\alpha} \cdot \mu$.

CASE 1. Let $\lambda_{i} \notin\left\{\mu, s_{\alpha} \cdot \mu\right\}$. In this case both Hom-spaces in (3) are zero by block decomposition and the claim is true.

CASE 2. Let $\lambda_{i}=s_{\alpha} \cdot \mu>\mu$. In this case we get $P_{R_{\mathfrak{p}}}\left(\lambda_{i}\right) \cong \Delta_{R_{\mathrm{p}}}\left(\lambda_{i}\right) \neq$ $\Delta_{R_{p}}(\mu) \cong \nabla_{R_{p}}(\mu)$ and therefore

$$
\begin{aligned}
& \operatorname{Hom}_{\mathscr{O}_{R_{\mathrm{p}}}}\left(P_{R_{\mathrm{p}}}\left(\lambda_{i}\right), \Delta_{R_{\mathrm{p}}}(\mu)\right)=\operatorname{Hom}_{\mathscr{O}_{R_{\mathrm{p}}}}\left(P_{R_{\mathrm{p}}}\left(\lambda_{i}\right), \nabla_{R_{\mathrm{p}}}(\mu)\right) \\
& =\operatorname{Hom}_{O_{R_{\mathrm{p}}}}\left(t\left(\Delta_{R_{\mathrm{p}}}(\mu)\right), t\left(P_{R_{\mathrm{p}}}\left(\lambda_{i}\right)\right)\right) \\
& =\left(\operatorname{Hom}_{\mathcal{O}_{R_{\mathrm{p}}}}\left(t\left(P_{R_{\mathrm{p}}}\left(\lambda_{i}\right)\right), \nabla_{R_{\mathrm{p}}}\left(\mu^{\prime}\right)\right)\right)^{*}=0
\end{aligned}
$$

and we are done.

CASE 3. Let $\lambda_{i}=\mu<s_{\alpha} \cdot \mu$. Then we have $\Delta_{R_{\mathrm{p}}}(\mu)=\nabla_{R_{\mathrm{p}}}(\mu)$ and also $t\left(P_{R_{\mathrm{p}}}\left(\lambda_{i}\right)\right) \cong K_{R_{\mathrm{p}}}\left(-2 \rho-\lambda_{i}\right) \cong P_{R_{\mathrm{p}}}\left(-2 \rho-s_{\alpha} \cdot \mu\right)$. With these assumptions on $\lambda_{i}$ and $\mu$, we get an injection

$$
\varphi: t\left(\Delta_{\mathrm{K}}(\mu)\right) \hookrightarrow t\left(P_{\mathrm{K}}\left(\lambda_{i}\right)\right)
$$

Since $-2 \rho-s_{\alpha} \cdot \mu<-2 \rho-\mu$, the map $\varphi$ is an embedding from a Verma module with dominant highest weight to the projective cover of the simple module with antidominant highest weight. These modules are objects of a subgeneric block of $\mathscr{O}_{\mathrm{K}}$ and this projective cover is self-dual. So dualizing $\varphi$ leads to a surjection 
$d \varphi: P_{\mathrm{K}}\left(-2 \rho-s_{\alpha} \cdot \mu\right) \rightarrow \nabla_{\mathrm{K}}(-2 \rho-\mu)$. But the composition $d \varphi \circ \varphi$ is nonzero. Thus, we get $E_{\mathrm{K}}(\varphi) \neq 0$. Comparing dimensions over K, we get an isomorphism

$$
E_{\mathrm{K}}: \operatorname{Hom}_{\mathscr{O}_{\mathrm{K}}}\left(t\left(\Delta_{\mathrm{K}}(\mu)\right), t\left(P_{\mathrm{K}}\left(\lambda_{i}\right)\right)\right) \stackrel{\sim}{\longrightarrow}\left(\operatorname{Hom}_{\mathcal{O}_{\mathrm{K}}}\left(t\left(P_{\mathrm{K}}\left(\lambda_{i}\right)\right), \nabla_{\mathrm{K}}\left(\mu^{\prime}\right)\right)\right)^{*}
$$

By Nakayama's lemma, we conclude that $E_{R_{\mathrm{v}}}$ is surjective and therefore an isomorphism.

We also have a surjective map $P_{\mathrm{K}}\left(\lambda_{i}\right) \rightarrow \Delta_{\mathrm{K}}(\mu)$ and with similar arguments we get an isomorphism of vector spaces

$$
\operatorname{Hom}_{\mathscr{O}_{\mathrm{K}}}\left(P_{\mathrm{K}}\left(\lambda_{i}\right), \Delta_{\mathrm{K}}(\mu)\right) \stackrel{\sim}{\longrightarrow} \operatorname{Hom}_{\mathscr{O}_{\mathrm{K}}}\left(P_{\mathrm{K}}\left(\lambda_{i}\right), \nabla_{\mathrm{K}}(\mu)\right)
$$

and Nakayama's lemma finishes this case.

CASE 4. Let $\lambda_{i}=\mu>s_{\alpha} \cdot \mu$. Then we have $P_{R_{\mathrm{p}}}\left(\lambda_{i}\right)=\Delta_{R_{\mathrm{p}}}\left(\lambda_{i}\right)=\Delta_{R_{\mathrm{p}}}(\mu)$ and over the residue field $\mathrm{K}$ we get $E_{\mathrm{K}}(\mathrm{id}) \neq 0$ and $J_{\mathrm{K}}(\mathrm{id}) \neq 0$. And again by Nakayama's lemma we get two isomorpisms

$$
\begin{array}{ll}
J: \operatorname{Hom}_{\mathscr{O}_{R_{p}}}\left(P_{R_{p}}\left(\lambda_{i}\right), \Delta_{R_{p}}(\mu)\right) & \stackrel{\sim}{\longrightarrow} \operatorname{Hom}_{\mathscr{O}_{R_{p}}}\left(P_{R_{p}}\left(\lambda_{i}\right), \nabla_{R_{p}}(\mu)\right) \\
E: \operatorname{Hom}_{\mathscr{O}_{R_{p}}}\left(t\left(\Delta_{R_{p}}(\mu)\right), t\left(P_{R_{p}}\left(\lambda_{i}\right)\right)\right) \stackrel{\sim}{\longrightarrow}\left(\operatorname{Hom}_{\mathscr{O}_{R_{p}}}\left(t\left(P_{R_{p}}\left(\lambda_{i}\right)\right), \nabla_{R_{p}}\left(\mu^{\prime}\right)\right)\right)^{*_{R_{p}}}
\end{array}
$$

and the claim is true.

CASE 5. Let $\lambda_{i}=s_{\alpha} \cdot \mu<\mu$. By the following two lemmas 4.6 and 4.7 we also get that the restriction of $L_{Q}$ to the $R_{\mathfrak{p}}$-lattice induces an isomorphism in this case and the proof is finished.

For the following two lemmas let $\mathfrak{p}=R \check{\alpha}$ and $\{\lambda, \mu\}$ be an equivalence class under $\sim_{R_{p}}$ with $\lambda=s_{\alpha} \cdot \mu<\mu$ and set $\lambda^{\prime}=-2 \rho-\lambda$.

Lemma 4.6. The map

$$
J_{R_{\mathrm{p}}}: \operatorname{Hom}_{\mathscr{O}_{R_{\mathrm{p}}}}\left(P_{R_{\mathrm{p}}}(\lambda), \Delta_{R_{\mathrm{p}}}(\mu)\right) \hookrightarrow \operatorname{Hom}_{\mathcal{O}_{R_{\mathrm{p}}}}\left(P_{R_{\mathrm{p}}}(\lambda), \nabla_{R_{\mathrm{p}}}(\mu)\right)
$$

has image $\check{\alpha} \cdot \operatorname{Hom}_{\mathcal{O}_{R_{\mathfrak{p}}}}\left(P_{R_{\mathfrak{p}}}(\lambda), \nabla_{R_{\mathfrak{p}}}(\mu)\right)$.

Proof. After base change with the residue field $\mathrm{K}$ we get

$$
J_{\mathrm{K}}: \operatorname{Hom}_{\mathscr{O}_{\mathrm{K}}}\left(P_{\mathrm{K}}(\lambda), \Delta_{\mathrm{K}}(\mu)\right) \longrightarrow \operatorname{Hom}_{\mathcal{O}_{\mathrm{K}}}\left(P_{\mathrm{K}}(\lambda), \nabla_{\mathrm{K}}(\mu)\right)
$$

On the left side we have the generator $P_{\mathrm{K}}(\lambda) \rightarrow \Delta_{\mathrm{K}}(\lambda) \hookrightarrow \Delta_{\mathrm{K}}(\mu)$ which becomes

$$
P_{\mathrm{K}}(\lambda) \rightarrow \Delta_{\mathrm{K}}(\lambda) \hookrightarrow \Delta_{\mathrm{K}}(\mu) \rightarrow L_{\mathrm{K}}(\mu) \hookrightarrow \nabla_{\mathrm{K}}(\mu)
$$


on the right side. But since this map is zero we get

$$
J\left(\operatorname{Hom}_{O_{R_{\mathrm{p}}}}\left(P_{R_{\mathrm{p}}}(\lambda), \Delta_{R_{\mathrm{p}}}(\mu)\right)\right) \subset \check{\alpha} \cdot \operatorname{Hom}_{\mathcal{O}_{R_{\mathrm{p}}}}\left(P_{R_{\mathrm{p}}}(\lambda), \nabla_{R_{\mathrm{p}}}(\mu)\right)
$$

Now we can lift the generator $j^{\prime}: P_{\mathrm{K}}(\lambda) \rightarrow \Delta_{\mathrm{K}}(\lambda) \hookrightarrow \Delta_{\mathrm{K}}(\mu)$ to a generator $j: P_{R_{\mathrm{p}}}(\lambda) \rightarrow \Delta_{R_{\mathrm{p}}}(\mu)$ of the $R_{\mathfrak{p}}$-module $\operatorname{Hom}_{\mathcal{O}_{R_{\mathrm{p}}}}\left(P_{R_{\mathrm{p}}}(\lambda), \Delta_{R_{\mathrm{p}}}(\mu)\right)$ via base change. Let also $l \in \operatorname{Hom}_{\mathcal{O}_{R_{\mathrm{p}}}}\left(P_{R_{\mathrm{p}}}(\lambda), \nabla_{R_{\mathrm{p}}}(\mu)\right)$ be a generator. Then we conclude $J_{R_{\mathfrak{p}}}(j)=c l$ for one $c \in R_{\mathfrak{p}}$ with $c \neq 0$. Multiplying with an appropriate invertible element of $R_{\mathfrak{p}}$ we can assume $c=(\check{\alpha})^{m}$ for $m \in \mathrm{N}$.

On $\Delta_{R_{\mathrm{p}}}(\mu)$ we have the filtration which comes from the preimages of $(\check{\alpha})^{i} \nabla_{R_{\mathfrak{p}}}(\mu)$ under the inclusion $\Delta_{R_{\mathfrak{p}}}(\mu) \hookrightarrow \nabla_{R_{\mathfrak{p}}}(\mu)$. Denote by $\Delta_{\mathrm{K}}(\mu)_{i}$ the image of the i-th filtration layer under the surjection $\Delta_{R_{\mathrm{v}}}(\mu) \rightarrow \Delta_{\mathrm{K}}(\mu)$ which is the $\mathrm{i}$-th layer of the Jantzen filtration on $\Delta_{K}(\mu)$. By the Jantzen sum formula we get

$$
\sum_{i>0} \operatorname{ch} \Delta_{\mathrm{K}}(\mu)_{i}=\sum_{\substack{\alpha \in \mathscr{R}^{+}, n \in \mathrm{N} \\\langle\mu+\rho+\tau, \alpha)_{\mathrm{K}}=n}} \operatorname{ch} \Delta_{\mathrm{K}}(\mu-n \alpha)=\operatorname{ch} \Delta_{\mathrm{K}}\left(s_{\alpha} \cdot \mu\right)
$$

Since $\Delta_{\mathrm{K}}\left(s_{\alpha} \cdot \mu\right)$ is simple, we conclude $\Delta_{\mathrm{K}}(\mu)_{1} \cong \Delta_{\mathrm{K}}\left(s_{\alpha} \cdot \mu\right)$ and $\Delta_{\mathrm{K}}(\mu)_{2}=0$.

If $J_{R_{\mathrm{p}}}(j) \in(\check{\alpha})^{2} \cdot \operatorname{Hom}_{\mathscr{O}_{R_{\mathrm{p}}}}\left(P_{R_{\mathrm{p}}}(\lambda), \nabla_{R_{\mathrm{p}}}(\mu)\right)$ we would get $j^{\prime}\left(P_{\mathrm{K}}(\lambda)\right) \subset$ $\Delta_{\mathrm{K}}(\mu)_{2}=0$, i.e., $j^{\prime}=0$, which is a contradiction to the choice of $j^{\prime}$. We conclude $J_{R_{\mathfrak{p}}}(j)=\check{\alpha} l$.

LeMma 4.7. The map

$$
E_{R_{\mathrm{p}}}: \operatorname{Hom}_{\mathcal{O}_{R_{\mathrm{p}}}}\left(t\left(\Delta_{R_{\mathrm{p}}}(\mu)\right), t\left(P_{R_{\mathrm{p}}}(\lambda)\right)\right) \hookrightarrow\left(\operatorname{Hom}_{\mathcal{O}_{R_{\mathrm{p}}}}\left(t\left(P_{R_{\mathrm{p}}}(\lambda)\right), \nabla_{R_{\mathrm{p}}}\left(\mu^{\prime}\right)\right)\right)^{*_{R_{\mathrm{p}}}}
$$

has image $\check{\alpha} \cdot\left(\operatorname{Hom}_{\mathcal{O}_{R_{\mathfrak{p}}}}\left(t\left(P_{R_{\mathfrak{p}}}(\lambda)\right), \nabla_{R_{\mathfrak{p}}}\left(\mu^{\prime}\right)\right)\right)^{*_{R_{\mathfrak{p}}}}$.

Proof. We have $t_{R_{\mathrm{p}}}\left(P_{R_{\mathrm{p}}}(\lambda)\right)=K_{R_{\mathrm{p}}}\left(\lambda^{\prime}\right)$ and by our assumptions we get $s_{\alpha} \cdot\left(\lambda^{\prime}\right)=\mu^{\prime}<\lambda^{\prime}$. But since $\mu^{\prime}$ is minimal in its equivalence class under $\sim_{R_{\mathrm{p}}}$, we get an isomorphism $P_{\mathrm{K}}\left(\mu^{\prime}\right) \cong K_{\mathrm{K}}\left(\lambda^{\prime}\right)$ and then an inclusion $\gamma^{\prime}$ : $\Delta_{\mathrm{K}}\left(\mu^{\prime}\right) \hookrightarrow K_{\mathrm{K}}\left(\lambda^{\prime}\right)$. Lifting this map with base change 2.2, we get a basis $\gamma$ of the $R_{\mathfrak{p}}$-module $\operatorname{Hom}_{\mathcal{O}_{R_{\mathrm{p}}}}\left(\Delta_{R_{\mathrm{p}}}\left(\mu^{\prime}\right), K_{R_{\mathrm{p}}}\left(\lambda^{\prime}\right)\right)$. But every map $\Delta_{\mathrm{K}}\left(\mu^{\prime}\right) \rightarrow \nabla_{\mathrm{K}}\left(\mu^{\prime}\right)$ which factors through $K_{\mathrm{K}}\left(\lambda^{\prime}\right)$ is zero already. So we conclude that

$$
E_{\mathrm{K}}: \operatorname{Hom}_{\mathscr{O}_{\mathrm{K}}}\left(\Delta_{\mathrm{K}}\left(\mu^{\prime}\right), K_{\mathrm{K}}\left(\lambda^{\prime}\right)\right) \longrightarrow\left(\operatorname{Hom}_{\mathscr{O}_{\mathrm{K}}}\left(K_{\mathrm{K}}\left(\lambda^{\prime}\right), \nabla_{\mathrm{K}}\left(\mu^{\prime}\right)\right)\right)^{*}
$$

is the zero map. If we now choose a basis $\delta$ of the free one dimensional $R_{\mathfrak{p}}$-module $\left(\operatorname{Hom}_{\mathcal{O}_{R_{\mathfrak{p}}}}\left(K_{R_{\mathfrak{p}}}\left(\lambda^{\prime}\right), \nabla_{R_{\mathrm{p}}}\left(\mu^{\prime}\right)\right)\right)^{*}$ and eventually multiply it with an appropriate invertible element of $R_{\mathfrak{p}}$, we conclude $E_{R_{\mathfrak{p}}}(\gamma)=(\check{\alpha})^{n} \delta$ for $n>0$. 
By Proposition 2.12 we get

$$
\begin{aligned}
\operatorname{End}_{\mathscr{O}_{R_{\mathfrak{p}}}}\left(K_{R_{\mathfrak{p}}}\left(\lambda^{\prime}\right)\right) & \cong \operatorname{End}_{\mathscr{O}_{R_{\mathfrak{p}}}}\left(P_{R_{\mathfrak{p}}}\left(\mu^{\prime}\right)\right) \\
& \cong\left\{(x, y) \in R_{\mathfrak{p}} \oplus R_{\mathfrak{p}} \mid x \equiv y \bmod \check{\alpha}\right\}
\end{aligned}
$$

where for $\varphi \in \operatorname{End}_{\mathcal{O}_{R_{\mathrm{p}}}}\left(K_{R_{\mathrm{p}}}\left(\lambda^{\prime}\right)\right) x$ and $y$ are given by the induced maps on the short exact sequences

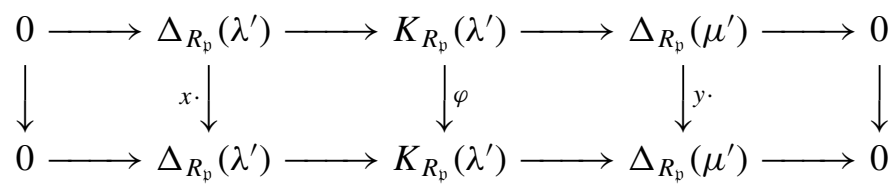

If for $\varphi$ we choose the homomorphism that corresponds to the tupel $(x, y)=$ $(0, \check{\alpha})$, the map on the cokernels of the diagram will factor through the middle and we get a map

$$
\Delta_{R_{\mathrm{p}}}\left(\mu^{\prime}\right) \rightarrow K_{R_{\mathrm{p}}}\left(\lambda^{\prime}\right) \longrightarrow \Delta_{R_{\mathrm{p}}}\left(\mu^{\prime}\right)=\nabla_{R_{\mathrm{p}}}\left(\mu^{\prime}\right)
$$

and the image of this map is $\check{\alpha} \nabla_{R_{\mathrm{p}}}\left(\mu^{\prime}\right)$. So we get $n=1$.

Remark 4.8. The last corollary shows the connection between the Jantzen and Andersen filtrations. Taking a projective generator of the block containing the Verma module $\Delta(\mu)$, the above filtration on the C-vector space $\operatorname{Hom}_{\mathfrak{g}}(P, \Delta(\mu))$ might carry enough information to get back the Jantzen filtration on $\Delta(\mu)$. If the map $t$ between the two Hom-spaces induced by the tilting functor also respects the gradings coming from the graded version of category $\mathscr{O}$, the results of [11] about the Andersen filtration could give an alternative proof of the Jantzen conjecture about the semisimplicity of the subquotients of the Jantzen filtration which was proved in [2].

\section{REFERENCES}

1. Arkhipov, S., Semi-infinite cohomology of associative algebras and bar duality, Internat. Math. Res. Notices (1997:17), 833-863.

2. Beilinson, A., and Bernstein, J., A proof of Jantzen conjectures, pp. 1-50 in: I. M. Gelfand Seminar, Adv. Soviet Math. 16:1, Amer. Math. Soc., Providence, RI 1993.

3. Beilinson, A., Ginzburg, V., and Soergel, W., Koszul duality patterns in representation theory, J. Amer. Math. Soc. 9 (1996), 473-527.

4. Collingwood, D., and Irving, R., A decomposition theorem for certain self dual modules in the category $\mathcal{O}$, Duke Math. J. 58 (1989), 89-102.

5. Fiebig, P., Centers and translation functors for the category $\mathcal{O}$ over Kac-Moody algebras, Math. Z. 243 (2003), 689-717.

6. Fiebig, P., The combinatorics of category $\mathscr{O}$ over symmetrizable Kac-Moody algebras, Transform. Groups 11 (2006), 29-49. 
7. Jantzen, J. C., Moduln mit einem höchsten Gewicht, Lecture Notes in Math. 750, Springer, Berlin 1979.

8. Soergel, W., Kategorie $\mathscr{O}$, perverse Garben und Moduln über den Koinvarianten zur Weylgruppe, J. Amer. Math. Soc. 3 (1990), 421-445.

9. Soergel, W., Character formulas for tilting modules over quantum groups at roots of one, pp. 161-172 in: Current Developments in Mathematics 1997, Proc. Cambridge, MA, Int. Press, Boston 1999.

10. Soergel, W., Character formulas for tilting modules over Kac-Moody algebras, Represent. Theory 2 (1998), 432-448.

11. Soergel, W., Andersen-Filtration and hard Lefschetz, Geom. Funct. Anal. 17 (2008), 20662089.

DEPARTMENT MATHEMATIK

UNIVERSITÄT ERLANGEN

CAUERSTR. 11

91058 ERLANGEN

GERMANY

E-mail: kuebel@mi.uni-erlangen.de 